\title{
Characterization of the nanophase precipitation in a metastable beta titanium-based alloy by electrical resistivity, dilatometry and neutron diffraction
}

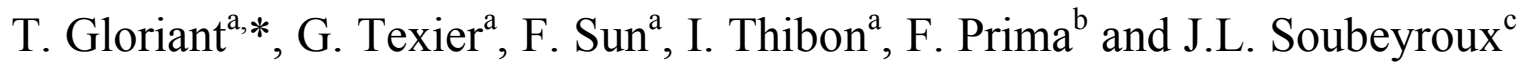
aINSA Rennes, UMR CNRS 6226 Sciences Chimiques de Rennes / Chimie-Métallurgie, 20 avenue des Buttes de Coësmes, F-35043 Rennes, France ${ }^{b}$ ENSC Paris, UMR CNRS 7045 Laboratoire de Physico-Chimie des Surfaces, 11 rue Pierre et Marie Curie, F-75231 Paris, France

'Institut Néel / CRETA, CNRS Grenoble, 25 avenue des Martyrs, F-38402 Grenoble, France

*Corresponding author.e-mail address: Thierry.Gloriant@insa-rennes.fr

Abstract: The metastable beta Ti-6Mo-5Ta-4Fe (wt.\%) alloys was synthesized by cold crucible levitation melting and then quenched in water from the beta phase field. In order to investigate the transformation sequence upon heating, thermal analysis methods such as electrical resistivity, dilatometry and neutron thermodiffraction were employed. By these methods, the different temperatures of transition were detected and solute partitioning was oberved to the beta matrix during the omega and alpha nanophase precipitation.

Keywords: titanium, phase transformations, neutron diffraction, electrical resistivity, dilatometry 
Over the last few decades, beta Ti alloys have continued on an increasing basis to be a very important class of materials because of their unique combination of high strength, excellent hardenability, low density and good corrosion resistance [1]. It has been shown that in most beta $\mathrm{Ti}$ alloys, an homogeneous nucleation and subsequent growth of finely dispersed intragranular alpha phase was accompanied by considerable strengthening and general increase of the mechanical properties (strength and toughness are enhanced while maintaining ductility to an acceptable level) [2]. The persistent problem is that $\alpha$ phase generally appears preferentially at grain boundaries, on intragranular defects and along dislocations lines. So that, it is rather difficult to obtain uniform and dispersed $\alpha$ phase distribution. Nevertheless, in metastable $\beta$ Ti alloys (obtained by quenching the alloy from the $\beta$ phase field), the precipitation of nanoscale $\omega$ phase particles is observed upon heating in Ti-based alloys containing the $\mathrm{Fe}, \mathrm{Mo}, \mathrm{Cr}$ and/or V $\beta$-stabilizer elements [3-5]. This additional phase has been widely observed to act as an heterogeneous nucleation site for $\alpha$ phase [6,7]. By this quenching/heating treatment, uniform and finer distribution of $\alpha$ precipitates in the $\beta$ matrix was obtained with the Ti-15V-3Cr-3Sn-3Al [8] and the « LCB » Ti-6.8Mo-4.5Fe-1.5Al alloy compositions [9] for example.

We have recently investigated the $\beta$ phase decomposition of new Ti-Ta-Mo and Ti-Ta-Mo-Fe metastable $\beta$ alloys by employing electric resistivity and differential scanning calorimetry [10]. We showed that the $\omega$ and $\alpha$ nanophase transitions could be well detected upon heating, particularly by use of the electrical resistivity measurement technique, which is very sensitive to minor constitutional changes produced by quenching, ageing and precipitation $[11,12]$. In the present study, an investigation of the transformation sequence by additional techniques such as dilatometry and neutron diffraction is proposed. These methods have never been used until now to characterize the nanophase formation upon heating from metastable $\beta$ Ti-based alloys. The aim of such additional characterization is to valid the resistivity measurements 
and also to try to elucidate the role of the solute elements during the $\omega$ and $\alpha$ nanophase precipitation. Results concerning the metastable $\beta$ Ti-6Mo-5Ta-4Fe alloy (wt.\%) are presented in this paper.

The Ti-6Mo-5Ta-4Fe alloy (wt.\%) was synthesized by the cold crucible levitation melting (CCLM) technique. The melting was carried out under a pure Ar atmosphere, which was introduced after several cycles of high vacuum pumping, because of the high reactivity of titanium with both oxygen and nitrogen. Notable features of CCLM are that it can melt metals with a high melting point, create an alloy of uniform composition and allow metals to be melted without crucible contamination. The melted alloy was cast into a cylindrical copper mould by removing the copper cold finger situated at the bottom of the cold crucible. Once the generator switched off, the melt drops and a cylindrical ingot with a diameter of $8 \mathrm{~mm}$ and about $10 \mathrm{~mm}$ in length was obtained. The ingot was solution treated at $850^{\circ} \mathrm{C}$ under high vacuum $\left(10^{-6}\right.$ mbar $)$ for one hour into a tubular furnace and then quenched in water at room temperature. The purpose of this heat treatment is to produce the desired homogenous $\beta$ microstructure alloy with $\beta$-stabilizer elements Ta, Mo and Fe.

The transformation sequence of the metastable $\beta$ titanium alloy was characterized upon heating by electrical resistivity measurement and by dilatometry. The electrical resistivity variation of the sample was carried out under high vacuum $\left(10^{-6}-10^{-7} \mathrm{mbar}\right)$ by using a serial electrical circuit set-up composed of the sample (with resistivity $\rho$ ), a reference resistor Rref (2 ohm) and a d.c. source (home-made apparatus). If we neglect the thermal expansion of the sample, the electrical resistivity ratio $\rho / \rho_{0}$ or the resistance ratio $R / R_{0}\left(R_{0}\right.$ : initial resistance at room temperature) plotted as a function of temperature (heating rate of $5^{\circ} \mathrm{C} / \mathrm{min}$ ) shows the structural transformations from room temperature to $900^{\circ} \mathrm{C}$. The dilatometry variation $\Delta \mathrm{l} / \mathrm{l}_{0}$ (home-made apparatus) of the sample was obtained during continuous heating carried out 
under high vacuum $\left(10^{-6}-10^{-7} \mathrm{mbar}\right)$ with a scan rate of $5^{\circ} \mathrm{C} / \mathrm{min}$ from room temperature to $900^{\circ} \mathrm{C}$.

The neutron thermodiffraction experiments were performed on the D1B two-axes diffractometer at the Institut Laue-Langevin, Grenoble, France. The neutron spectra were collected placing the 400 cells multidetector in a cylindrical geometry centered at the sample. The angular span was $80^{\circ}$, with steps of $0.2^{\circ}$, and a 2-theta starting angle of $40^{\circ}$. A graphite monochromator (002) was used, producing a $\lambda=2.52 \AA$ wavelength. The sample was heated from the room temperature to $900^{\circ} \mathrm{C}$ (heating rate: $3^{\circ} \mathrm{C} / \mathrm{min}$ ) in a furnace working under a vacuum of $10^{-4}-10^{-5}$ mbar. The neutron flux on the sample was of about $10^{6} \mathrm{n} /\left(\mathrm{s} \mathrm{cm}^{2}\right)$, which allowed us to monitor the evolution during heating by diffraction patterns collected every 5 min. Finally, the samples were naturally cooled down to the room temperature.

For transmission electron microscopy (TEM) observations, thin foil samples were obtained by mechanical grinding to $200 \mu \mathrm{m}$ and twin jet electropolishing (6\% perchloric acid in acetic acid; $22 \mathrm{~V}$ ). Conventional TEM observations were carried out using a JEOL 120CX II Electron Microscope operating at $120 \mathrm{kV}$.

In order to investigate the phase transformation sequence from the $\beta$ as-quenched state, the alloy was first characterized upon continuous heating (heating rate of $5^{\circ} \mathrm{C} / \mathrm{min}$ ) by electrical resistivity and dilatometry measurements. Figure 1 shows the electrical resistivity (in grey) and the dilatometry (in black) variation curves for the Ti-6Mo-5Ta-4Fe alloy.

It has already been reported that the negative temperature dependence observed on the resistivity curve at low temperature is due to the presence of the athermal omega phase $\left(\omega_{\text {ath }}\right)$, which is formed during quenching and disappears reversibly upon heating $[3,12,13]$. A (111) $\beta$ plane collapse model has been proposed to explain the $\beta / \omega_{\text {ath }}$ displacive transformation: the $\omega$ lattice can be obtained by collapsing one pair of (111) planes to the intermediate position 
leaving the adjacent (111) planes unaltered. [14]. The interatomic distances of the quenched $\omega_{\text {ath }}$ phase have been recently characterized by neutron diffraction in the Ti-V system [15].

At higher temperature of heating, $\omega_{\text {iso }}$ hexagonal phase can precipitate irreversibly, implying diffusional phenomena. The formation of this $\omega_{\text {iso }}$ phase on heating is shown to occur at 260$280^{\circ} \mathrm{C}$ (identified by $\mathrm{T} \omega_{\text {iso }}$ in the figure 1 ) where an increase in the resistivity and a decrease in the dilatometry curves are observed simultaneously.

This $\omega_{\text {iso }}$ phase is nanometer in size and transmission electron microscopy is required for its observation. The bright-field TEM micrograph presented in figure 2 corresponds to the Ti$6 \mathrm{Mo}-5 \mathrm{Ta}-4 \mathrm{Fe}$ alloy, which was annealed at $300^{\circ} \mathrm{C}$ for 1 hour. The size of the observed $\omega_{\text {iso }}$ particles (in dark) is about $20-30 \mathrm{~nm}$, which is in good agreement with the size found in literature [6-10].

From $350^{\circ} \mathrm{C}$, the important resistivity drop observed (indicated by $\mathrm{T} \alpha_{\text {nano }}$ in the figure 1 ) is due to the progressive vanishing of $\omega_{\text {iso }}$ through a $\omega / \alpha$ phase transformation. From this temperature, $\omega_{\text {iso }}$ phase has been widely observed to act as an heterogeneous nucleation site for the $\alpha$ phase formation [6] and we showed in a recent paper [9] the nucleation of the $\alpha$ phase in the core of the $\omega_{\text {iso }}$ particles by high-resolution electron microscopy. By this heterogeneous nucleation mechanism, the $\alpha$ phase is observed to be finely dispersed in the $\beta$ matrix. The dark-field TEM image presented in the figure 3 shows the distribution of the $\alpha$ nanoprecipitates (in bright) in the Ti-6Mo-5Ta-4Fe alloy annealed at $400^{\circ} \mathrm{C}$ for 1 hour. It has been shown in literature that stable nanostructured alloys can be then obtained by a duplex ageing treatment. This treatment consists to form a maximum of $\omega_{\text {iso }}$ particles at low temperature ( $1^{\text {st }}$ step) and to complete the progressive vanishing of $\omega_{\text {iso }}$ into the $\alpha$ nanoprecipitates by heterogeneous nucleation mechanism at higher temperature $\left(2^{\text {nd }}\right.$ step) $[1,8,10]$ 
By heating the alloy at a higher temperature, precipitation of conventional inter and intra granular $\alpha$ phase, micrometer in size, occurs at about $560^{\circ} \mathrm{C}$ where a slight increase of electrical resistivity is observed ( $\mathrm{T} \alpha_{\mathrm{eq}}$ in the figure 1$)$. This temperature corresponds to the return towards the equilibrium $\alpha+\beta$ domain associated with the vertical dotted line in the figure 1 . This transformation is also clearly detected by dilatometry where a brush backward is observed on the curve for this temperature.

The results obtained in this study show that the phase transitions detected by dilatometry are in good accordance with those measured by electrical resistivity: both techniques appear very useful for the characterization of metastable $\beta$ Ti-based alloys. From the dilatometry curve, it could be estimated that the decrease observed at $\mathrm{T} \omega_{\text {iso }}$ is due to the fact that during the diffusion-controlled formation of $\omega_{\text {iso }}$, the $\beta$-stabilizer alloying elements are rejected to the surrounding $\beta$ matrix since the atomic radii of Fe $(1.26 \AA)$ and Mo $(1.39 \AA)$ are smaller than the atomic radius of $\mathrm{Ti}(1.47 \AA$ ) (atomic radius of Ta is closed to Ti at $1.49 \AA$ ).

Figure 4 shows the neutron diffraction pattern evolution of the Ti-6Mo-5Ta-4Fe alloy continuously heated up to $900^{\circ} \mathrm{C}$ (heating rate of $3^{\circ} \mathrm{C} / \mathrm{min}$ ) and then naturally cooled (from the black line) to the room temperature. On this figure, the neutron diffraction peaks corresponding to the $\beta$ phase and to the $\alpha$ phase are detected and indexed. The dotted line corresponds to the equilibrium $\alpha+\beta$ domain limit situated at about $560^{\circ} \mathrm{C}$. This temperature is in good accordance with those previously detected by resistivity and dilatometry (T $\alpha_{\text {eq }}$ in the figure 1). The $\alpha$ diffracted peaks are only observed above this temperature, which corresponds to the precipitation of conventional inter and intra granular $\alpha$ phase, micrometer in size. In the temperature range corresponding to the formation of $\omega_{\text {iso }}$ and $\alpha_{\text {nano }}$ phases (below the dotted line), only the peaks related to the $\beta$ matrix are observed, particularly the one related to the (110) plan. In fact the $\omega_{\text {iso }}$ and $\alpha_{\text {nano }}$ phases are too small to be detected by neutron diffraction. 
In the figure 5, we have reported the crystallographic cell parameter variation upon heating and cooling (black curve) and the diffraction intensity evolution (grey curve) upon heating concerning the $\beta(110)$ peak. It is observed on this figure a decreasing of the $\beta$ cell parameter intervening at about $270-280^{\circ} \mathrm{C}$. This observation confirms the dilatometry analysis: the decreasing of the $\beta$ cell parameter is induced by the diffusion of the alloying elements to the $\beta$ matrix during the formation of $\omega_{\text {iso }}$ at $\mathrm{T} \omega_{\text {iso. }}$. From the neutron diffraction analysis, the $\alpha$ nanophase precipitation can also be observed. This transition is detected at about $350^{\circ} \mathrm{C}$ on the black curve (indicated by $\mathrm{T} \alpha_{\text {nano }}$ ), where a second $\beta$ cell parameter decreases is observed. This temperature is in a good accordance with the one detected by electrical resistivity. It is surprising to observe an increase of the neutron diffraction intensity of the $\beta(110)$ peak below the dotted line as only this phase is detected at low temperature (grey curve in the figure 5). It is well known that the neutron diffraction intensity can be strongly influenced by the chemical composition. Consequently, the increase of the $\beta(110)$ peak is probably due to the enrichment of the alloying element in the $\beta$ matrix occurring during the $\omega$ and $\alpha$ nanophase precipitation. This intensity variation remains however complicated to evaluate in such nanostructured alloy composed of nanodomains alternatively rich in $\beta$-stabilizerelements (the $\beta$ matrix) and rich in the titanium element (the $\omega_{\text {iso }}$ and $\alpha$ nanoprecipitates). These nanodomains are probably not completely homogenous, particularly at the interfaces where the presence of a diffusion gradient is obvious. Nevertheless, we show here a clear experimental observation that the neutron diffraction intensity increase is due to the nanophase formation, in agreement with previous observations that solute partitioning occurs into the $\beta$ matrix during the $\omega_{\text {iso }}$ and $\alpha_{\text {nano }}$ precipitation.

In summary, this study shows that the electrical resistivity, the dilatometry and the neutron thermodiffraction techniques are very useful to characterize the metastable $\beta$ phase 
decomposition upon heating. In the quenched metastable beta Ti-6Mo-5Ta-4Fe (wt.\%) alloy, the $\omega_{\text {iso }}$ and $\alpha_{\text {nano }}$ nanophases were detected to precipitate at about $270^{\circ} \mathrm{C}$ and $350^{\circ} \mathrm{C}$, respectively and a solute partitioning was observed in the beta matrix during their diffusioncontrolled formations.

Acknowledgements: Neutron diffraction measurements were done in the framework of the CNRS D1B-Collaborating Research Group programme

\section{References}

[1] S. Ankem, C.A. Green, Mater. Sci. Eng. A263 (1999) 127.

[2] H. Nishizawa, E. Sudekai, W. Liu, H. Hashimoto, Mater. Trans. JIM 36 (1995) 924.

[3] J.C. Williams, M.J. Blackburn, Met. Soc. of AIME-Trans. 245 (1969) 2352.

[4] T. Yamane, A. Miyakubi, Proc. 4th World Conf. on Titanium, Met. Soc. of AIME 2 (1980) 1309

[5] U.M . Naik, R. Krishnan, Proc. 4th World Conf. on Titanium, Met. Soc. of AIME 2 (1980) 1335

[6] Y. Takemoto, M. Hida, A. Sakakibara, J. Japan Inst. Metals 57 (1993) 261.

[7] T.W. Duerig, G.T. Terlinde, J.C. Williams, Metall. Trans. A 11 (1980) 1987.

[8] T. Furuhara, T. Maki, T. Makino, J. Mater. Proc. Tech. 117 (2001) 318.

[9] F. Prima, P. Vermaut, G. Texier, D. Ansel, T. Gloriant, Scripta Mater. 54 (2006) 645.

[10] T. Gloriant, G. Texier, F. Prima, D. Laillé, D.M. Gordin, I. Thibon, D. Ansel, Adv. Eng. Mater. 8 (2006) 961.

[11] M.A. Hill, D.H. Polonis, J. Mater. Sci. 22 (1987) 2181.

[12] F. Prima, P. Vermaut, D. Ansel, J. Debuigne, Mater. Trans. JIM 41 ( 2000) 1092. 
[13] M. Ikeda, S.Y. Komatsu, T. Sugimoto, K. Kamei, Proc. 6th World Conf. on Titanium, in : P. Lacombe (Eds.), les Editions de Physique, Paris, 1988, p.313

[14] D. de Fontaine, Metall. Trans. A 19 (1988) 169.

[15] G. Aurelio, A. Fernández Guillermet, Scripta Mater. 43 (2000) 665.

\section{Figure captions :}

Figure 1. Electrical resistivity (grey curve) and dilatometry (black curve) variations of the metastable $\beta$ Ti-6Mo-5Ta-4Fe alloy upon heating (heating rate: $5^{\circ} \mathrm{C} / \mathrm{min}$ ).

Figure 2. Bright-field TEM image showing the $\omega_{\text {iso }}$ nanoprecipitates in the Ti-6Mo-5Ta-4Fe alloy annealed at $300^{\circ} \mathrm{C}$ for 1 hour.

Figure 3. Dark-field TEM image showing the $\alpha_{\text {nano }}$ nanoprecipitates in the Ti-6Mo-5Ta-4Fe alloy annealed at $400^{\circ} \mathrm{C}$ for 1 hour.

Figure 4. Neutron thermodiffraction pattern evolution of the metastable $\beta$ Ti-6Mo-5Ta-4Fe alloy upon heating (heating rate: $3^{\circ} \mathrm{C} / \mathrm{min}$ ).

Figure 5. Neutron diffraction intensity (grey curve) and crystallographic cell parameter (black curve) variations of the $\beta(110)$ peak upon heating (heating rate: $3^{\circ} \mathrm{C} / \mathrm{min}$ ). 


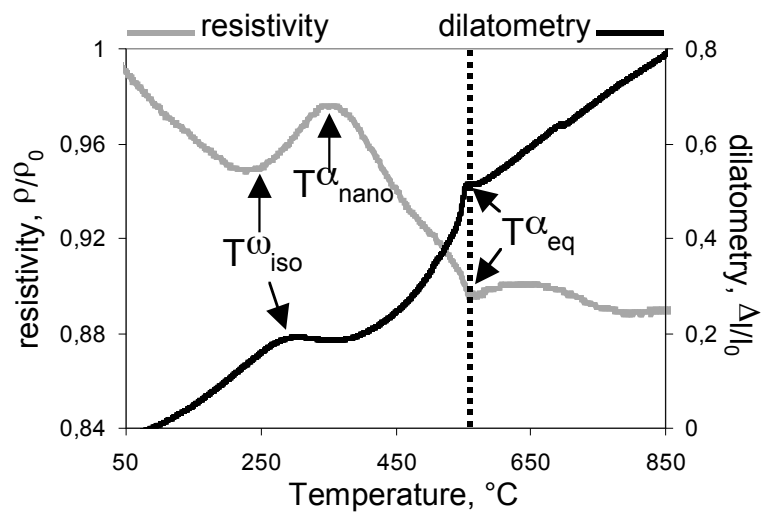

Figure 1 


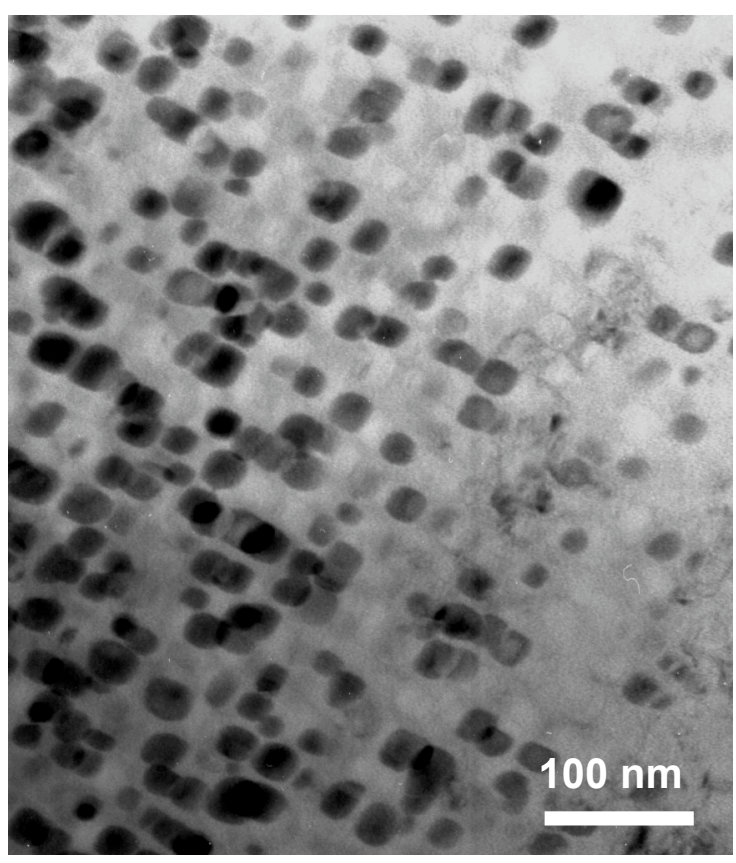

Figure 2 


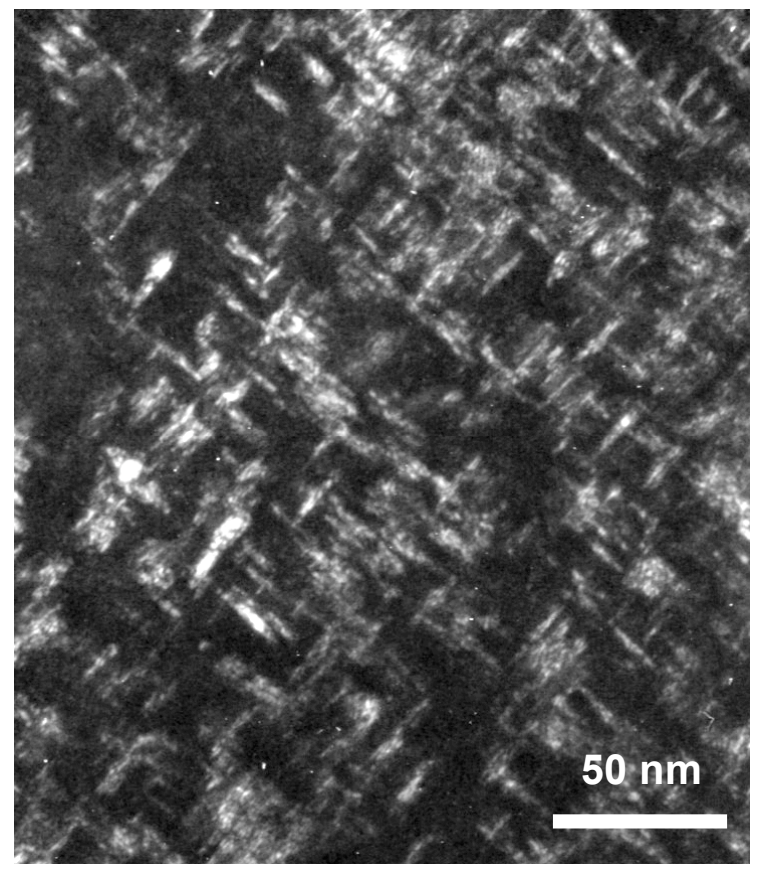

Figure 3 


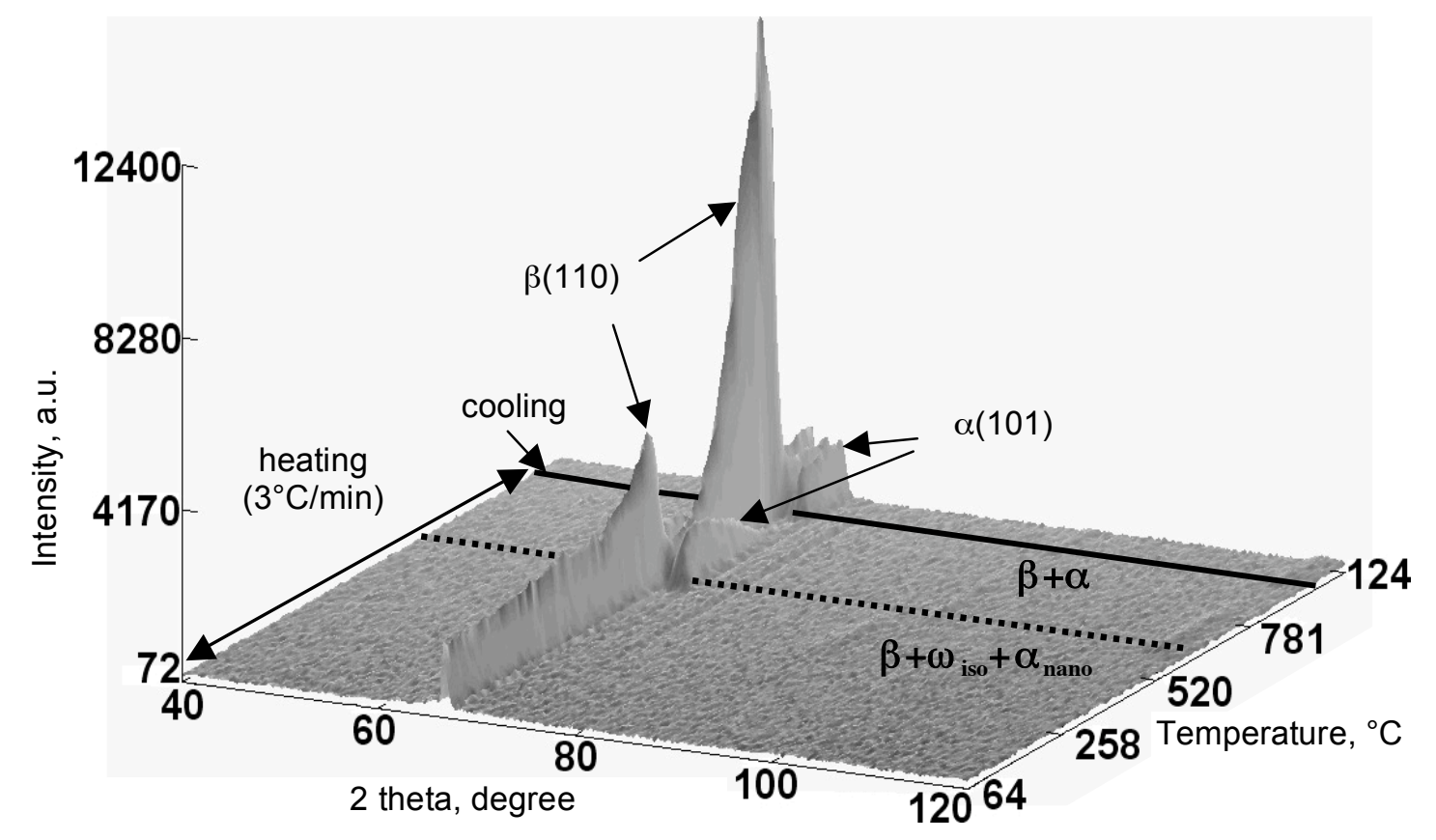

Figure 4 


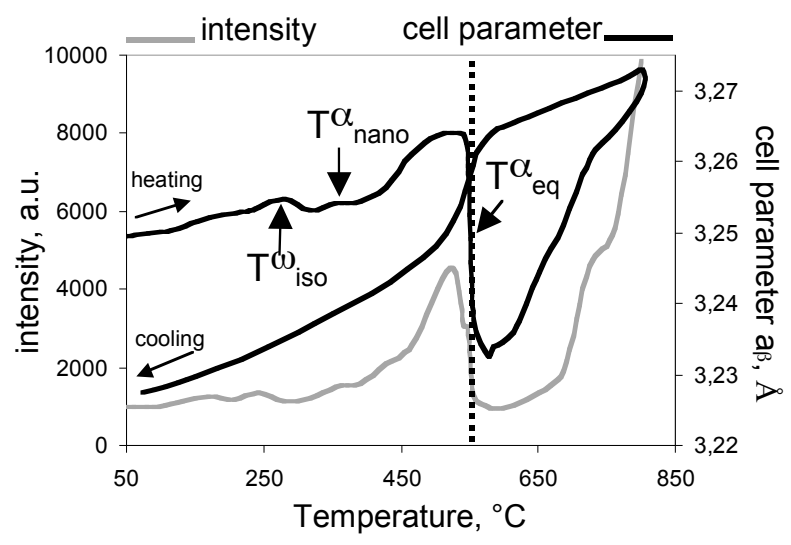

Figure 5 